\title{
Special Issue on Environmental Geostatistics
}

\author{
J. Jaime Gómez-Hernández • Celine Scheidt
}

Received: 31 May 2013 / Accepted: 6 June 2013 / Published online: 25 June 2013

(C) International Association for Mathematical Geosciences 2013

For many years geostatistics was constrained to mining applications, at a time when computing experimental variograms could require a pile of perforated cards and a day of computation to get the print out from a mainframe computer. As computational power increased, and the benefits of properly accounting for spatial heterogeneity were acknowledged, geostatistics moved into petroleum engineering, and soon into other disciplines like hydrogeology, soil science, ecology, epidemiology, environmental monitoring, agronomy or atmospheric sciences. In summary, geostatistics moved into any discipline that deals with spatial data.

Environmental engineers were not early adopters of geostatistics, but in the last decade, the use of geostatistics for environmental applications, as well as the development of techniques specifically targeted for environmental problems, has increased substantially. This special issue is a showcase for some recent work on what we could call environmental geostatistics.

The first paper by Davison, Huser, and Thibaud is a very interesting overview of current work on statistics of spatial extremes. It is a subject that has not attracted much attention in the geostatistical community but has clear applications in environmental sciences and engineering, particularly in these days of climate change analyses. After introducing a type of random function model that would be more appropriate than Gaussian models for the study of extreme data, the paper presents some applications for the analysis of winter minimum temperatures and extreme daily rainfall.

J.J. Gómez-Hernández (凶)

Research Institute of Water and Environmental Engineering, Universitat Politècnica de València, Valencia, Spain

e-mail: jgomez@upv.es

C. Scheidt

Department of Energy Resources Engineering, Stanford University, Stanford, CA, USA 
The second paper by Kyriakidis and Gaganis addresses the important problem of how to efficiently sample the space of uncertainty of a random function. Efficient sampling of uncorrelated random variables has been analyzed for a long time, but the problem of how to deal with correlated random variables is more demanding. Two such methods are proposed: latin hypercube sampling and stratified likelihood sampling. Both methods are demonstrated in the generation of unconditional realizations of hydraulic conductivities and shown to be more efficient than standard random sampling.

The third paper by De Iaco, Posa, and Palma tackles another complex problem, that of modeling vectorial fields in two dimensions; for this purpose, the authors propose the use of complex-valued random fields. The authors distinguish between vectorial fields and bivariate fields, and build on this difference to analyze the estimation and fitting of complex covariances, and the prediction of complex-valued random fields. The authors illustrate their findings with an application to the interpolation of wind speed.

The fourth paper by Horta, Correia, Pinheiro and Soares discusses an interesting approach for taking advantage of extensive geophysical sampling to generate conditional realizations of soil contamination in three dimensions. The authors use the two-dimensional information provided by the geophysical data with the threedimensional sample data in a clever iterative approach based on co-located direct sequential co-simulation to generate three-dimensional realizations of soil contamination. The method is illustrated in a field case study in Portugal.

The fifth paper by Jeannée, Bardou, Faucheux, and Ornstein studies the spatial distribution of ice content in the Bonnard glacier in Switzerland, which constitutes an environmental hazard. The main contribution of the paper is the demonstration of the pluri-Gaussian algorithm in a complex setting with real data. Given the strong relationship between lithology and ice content, the pluri-Gaussian algorithm is used to generate three-dimensional realizations of lithology respecting the expected facies transitions inferred from field survey and boreholes; then, the facies are populated with ice content data.

The sixth paper by Josset and Lunati addresses the issue of uncertainty quantification. Instead of applying a classical Monte Carlo analysis consisting of generating and evaluating hundreds of realizations using an accurate, time consuming numerical flow model, the authors propose to use a fast approximate model together with a clustering approach to select a number of realizations. The selected realizations are then used in the accurate model to estimate the uncertainty about the system. To account for potential bias in the approximate model, they introduce two error models: a local error model and a global model error. The local error model includes intracluster variability in the uncertainty model. The global error model employs a linear interpolation of the errors between the accurate and the approximate models at the realizations selected by clustering.

The final paper by Sapriza, Jódar, Carrera and Gupta, deals with climate change, global circulation models, and the need to generate daily spatially correlated rainfall fields. The challenges with daily rainfalls are to cope with the potential of having two different precipitation mechanisms, frontal and convective, and to incorporate the influence of orography. These two problems are dealt with by using discrete stochastic 
rainfall generating processes, each of which generates correlated rainfall fields associated with each season and different atmospheric circulation patterns. An application to the upper Guadiana basin in Spain demonstrates the method.

Environmental geostatistics has come a long way since the first applications in the late years of the past century, and is well deserving of its place in the mathematical geosciences. 